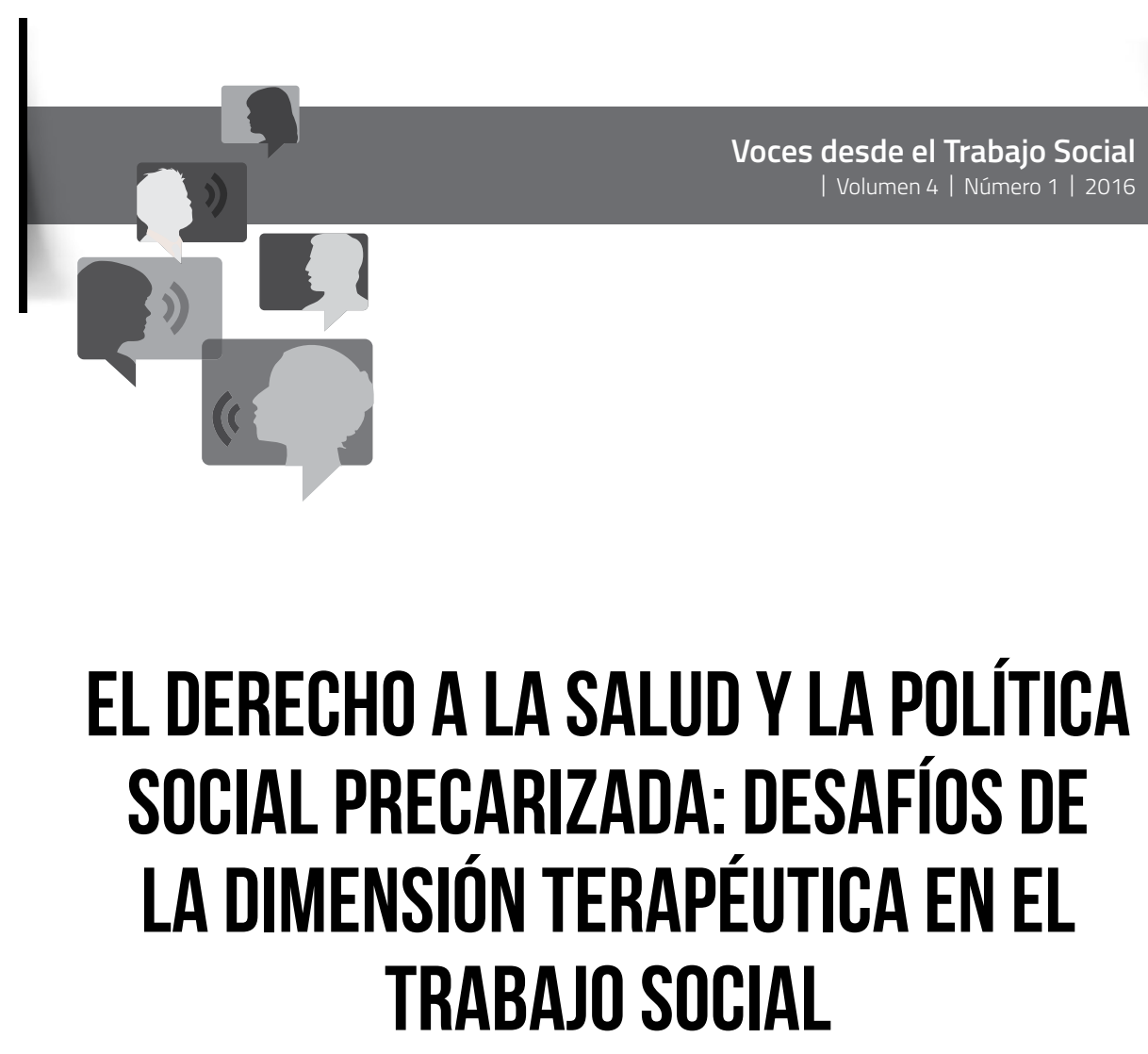




\section{Esterla Barreto Cortez}

Ha trabajo con mujeres sobrevivientes de violencia doméstica y sus hijos, programas de prevención para jóvenes y organización comunitaria. Es investigadora y ha evaluado programas sociales. Actualmente es catedrática de la Escuela Graduada de Trabajo Social de la Universidad de Puerto Rico, Recinto de Río Piedras.

\section{Nélida Rosario Rivera}

Egresada de la Escuela Graduada Beatriz Lassalle de la Universidad de Puerto Rico. Ha trabajado con jóvenes en el sistema de justicia. Laboró en la Procuraduría de Menores del Departamento de Justicia. Actualmente ofrece servicios al Colegio de Profesionales del Trabajo Social como Enlace con los Capítulos y Colegiados.

\section{Francine Sánchez Marcano}

Trabajadora Social con maestría en individuos y familias, de la Escuela Graduada Beatriz Lasalle de la Universidad de Puerto Rico en Río Piedras. Experiencia laboral en el escenario escolar y comunitario. Actualmente labora en la Compañía para el Desarrollo Integral de la Península de Cantera.

\section{Recibido:}

1 de marzo de 2016

\section{Aprobado:}

1 de septiembre de 2016 


\title{
EL DERECHO A LA SALUD Y LA POĹITICA SOCIAL PRECARIZADA: DESAFÍOS DE LA DIMENSIÓN TERAPÉUTICA EN EL TRABAJO SOCIAL
}

\author{
Esterla Barreto Cortez, Nélida Rosario Rivera \\ Francine Sánchez Marcano
}

Resumen

En Puerto Rico están ocurriendo una serie de cambios en el Trabajo Social (TS), entre ellos, la proliferación del TS Clínico (TSC) y relacionado a esta, la dimensión terapéutica en la profesión, lo cual amerita un análisis del contexto. En el presente trabajo proponemos que estos cambios están relacionados a la deslegitimación y ruptura del TS y de la funcionalidad que tiene el TSC para el Estado Neoliberal. El desmantelamiento del Estado Benefactor, comandado por el capital financiero, ha desplazado los derechos que deben ser garantizados por el Estado al mercado de consumo, que los vende a través de servicios ofrecidos por corporaciones privadas. Por esto, la política social se aleja de su universalidad, focalizándose y siendo precarizada para la clase trabajadora de bajos ingresos o desempleada. En el campo de la salud esto implica 
la trasformación de los servicios públicos a la compra a través de terceros pagadores de seguros de salud privados. Estos nuevos mecanismos de regulación del capital impulsan el ejercicio liberal de la profesión, el incremento en enfoques de autoayuda y 'coaching', y el marcado énfasis en la psicologización de la "cuestión social".

Descriptores: Trabajo Social Clínico, Salud Mental, Precarización laboral, Política Social, Derechos Humanos.

\section{Abstract}

In Puerto Rico there are a series of changes occurring in Social Work (SW), among these, the proliferation of Clinical Social Work (CSW), and related to this, the therapeutic dimension of the profession, which requires an analysis of the context. In this paper we propose that these changes are related to the delegitimization and rupture of Social Work, and the functionality that CSW offers to the Neoliberal State. The dismantling of the Welfare State, led by the financial capital, has shifted the human rights guaranteed by the State to the market, to be sold through services offered by the private sector. Thus, drawing social policy away from its universal character, to be focused and precarized for lowincome or unemployed working class populations. In the health field, it involves the transformation of public services to be purchased through private health insurance. These new capitalist regulatory 
mechanisms are driving the liberal practice of the profession, the increase in self-help approaches and coaching, and the strong emphasis on the psychologizing of the "social question".

Keywords: Clinical Social Work; Mental Health, labor conditions, Social Policy, Human Rights. 
Este trabajo ${ }^{1}$, se enfoca en la relación entre los cambios ocurridos en el campo de la salud mental a raíz de la transformación del sistema de salud pública en PR y sus vínculos a los cambios, las propuestas y los retos que estos representan para la dimensión clínica o terapéutica del TS. A partir de una compresión socio-histórica de la profesión sostenemos que hay una diferencia entre la dimensión o práctica clínica o terapéutica del TS y el TSC. Además, analizamos las implicaciones que la privatización de los servicios sociales y la proliferación del ejercicio autónomo y liberal de la profesión en el área clínica tienen sobre la concepción de los sujetos sociales como portadores de derechos o consumidores de servicios. Finalmente, discutimos algunos de los retos que esto representa para la consolidación del proyecto ético-político profesional y la formulación de una nueva ley reglamentadora de la profesión.

\section{Contexto socio-histórico}

Con la vuelta al liberalismo económico impuesto por la política neoliberal, (la cual se origina en la década del 70 , pero en PR se manifiesta de manera evidente en los 90 con el gobierno de Pedro Rosselló), el principal foco de intervención es desmantelar el Estado Benefactor lo que ha resultado en vulnerar los derechos adquiridos que a través de éste se

$1 \quad$ Este artículo parte de reflexiones iniciadas en el año 2011 a través del proceso de tesis de Nélida Rosario y Francine Sánchez, dirigido por Esterla Barreto. Posteriormente, se han dado encuentros académicos y profesionales junto a Mabel López, lo cual ha llevado a desarrollar un equipo de investigación que ha contado con la participación de los estudiantes doctorales, Jessenia Rivera y Eduardo Zavala en calidad de asistentes de investigación. El grupo de trabajo interesa estudiar los vínculos entre los derechos sociales, la política social y la profesión del TS. En particular analizar los cambios ocurridos en y entre estas, a partir de la hegemonía del capital financiero y la política neoliberal en Puerto Rico. 
confieren (Clínica Internacional de Derechos Humanos de la Facultad de Derecho de la Universidad Interamericana de Puerto Rico, CIDH, 2016). Esto ha implicado la privatización de los servicios sociales, la focalización y precarización de la política social, el encarecimiento de los servicios sociales públicos, el desplazamiento de los servicios sociales al mercado y la precarización de las condiciones de empleo de la clase trabajadora, incluyendo la clase profesional del TS. Tómese por ejemplo, entre otros, la Reforma de Salud que desmanteló el sistema de salud pública en el país para privatizar los servicios a través de un modelo de Cuidado Coordinado o Dirigido, (Rivera, Fernández, Torres y Parrilla, s/f; $\mathrm{CIDH}$, 2016); la privatización de los servicios de salud mental (Rivera, Fernández, Torres y Parrilla, s/f; Rivera, 2014; Fitzpatrick, 2013; CIDH, 2016; Gómez, 2014); la privatización de la administración de residenciales públicos (Cotto, 2007); la reestructuración de las escuela pública, así como los intentos de su privatización mediante la creación de escuelas chárter $(\mathrm{CIDH}, 2016$; Torres González, 2015); el ataque a la legislación laboral (Torres Rivera, 2011); y la privatización de otros segmentos de los servicios públicos para colocarlos bajo la administración de empresas privadas (Silva Gotay, s/f) y organizaciones sin fines de lucro (Díaz, López y Cruz, 2015; Pérez, 2015).

Por ende, aquellos derechos humanos, en particular los sociales, que constituían la base de políticas sociales universales y de buena calidad, a pesar de sus limitaciones y contradicciones, hoy están siendo recortados. Se ha generado la construcción de una ciudadanía de consumo, en la cual tienen derechos a servicios sociales de calidad aquellos que pueden pagarlos. Mientras, los grupos más vulnerados quedan a la merced de servicios de variada calidad ofrecidos desde el Estado o desde corporaciones, con y sin fines de lucro, subvencionadas con fondos del 
Estado. El desmantelamiento del Estado Benefactor propicia las condiciones para la expansión del mercado de la salud, la educación y la vivienda de calidad para quienes tienen los recursos económicos para costearlos, mientras se ofrecen servicios focalizados y de variada (y a veces dudosa) calidad para los sectores más empobrecidos y vulnerados, a través de agencias públicas, así como de empresas y organizaciones sin fines de lucro que se agrupan bajo un llamado "Tercer Sector" (CIDH, 2016; Díaz, López y Cruz, 2015; Pérez, 2015).

Por otra parte, se han psicologizado problemas cuyas raíces son inminentemente socio-estructurales en el orden del sistema-mundo capitalista/moderno-colonial, expandiendo así un complejo médico industrial cuyo interés primordial está en las ganancias que generan la medicación y ciertas terapias. Netto (1992) señala que las manifestaciones de la cuestión social propias del orden capitalista son refractadas como problemas sociales por los cuales se responsabiliza al sujeto singular por su destino personal. Con ello se refiere el proceso de individualización o psicologización de los problemas sociales cuya resolución es colocada en la modificación de la conducta de los individuos o el ajuste de su personalidad, en lugar de ser colocadas sobre la cuestión social. En el campo de la salud mental, nos referimos a situaciones como: la sobre-medicación de personas de mayor edad para mantenerlas controladas a falta de servicios de apoyo y cuido (El Nuevo Día, 14 de mayo de 2014); el etiquetar malestares del diario vivir con diagnósticos de salud mental (Gómez, 8 de noviembre de 2013); o los diagnósticos incorrectos de niños y niñas en el programa de educación especial del Departamento de Educación (Gómez, 28 de octubre de 2014). También se pudiera señalar el aumento en trastornos de ansiedad y depresivos, y otros causados por el uso de alcohol y las drogas, los cuales se asocian a conflictos sociales (Departamento de Salud, 2015). 
Iriart (2014) señala que con la mercantilización del derecho a la salud y la entrada del capital financiero a este mercado ocurre un proceso de biomedicalización en el cual las farmacéuticas desarrollan nuevas estrategias para aumentar el consumo y sus ganancias. La autora analiza cómo opera este proceso en el alarmante aumento del diagnóstico de TDHA,

Se transforma al sufrimiento infantil en un desbalance bioquímico de fácil diagnóstico, del que hay que estar alerta para controlarlo en base a medicamentos y/o tratamiento del niño, en lugar de repensar la situación de la infancia en el contexto del avance de procesos de transformación profunda de la vida a escala planetaria y de reconfiguraciones y disputas capitalistas. En lugar de ver las conductas disruptivas como un síntoma de un malestar social, se la ve como un problema biológico a ser tratado individualmente, muchas veces con fármacos con la iatrogenia que esto implica. (p. 11).

Para el TS situaciones como las descritas han representado un impacto en la proliferación del TSC, cuyos inicios para algunos se remonta al trabajo de casos propiciado a principios de Siglo XX por Mary Richmond (Northen, 1995). En el país, este ha tenido una vertiginosa proliferación a partir de la década del 70 en el contexto de la política neoliberal de privatización de los servicios sociales y la subsiguiente expansión del mercado de la salud en los 90. La dimensión clínica es una sobre la cual se debe promover mayor debate y discusión, pues se ha encontrado que no hay claridad con relación a lo que la caracteriza, la distingue de otras profesiones y sobre las implicaciones que tiene la psicologización de los problemas sociales para una profesión que se fundamenta en los derechos sociales y en la acción sobre lo social (Rosario \& Sánchez, 2012). 
Para el profesional y la profesional del TS todo lo anterior ha representado: (a) el empeoramiento de sus condiciones de trabajo; (b) el cambio de puestos de TS por otras nomenclaturas, aumentado así la competencia con otras profesiones de la conducta y voluntarios legos2; (c) una significativa concentración de profesionales especializándose en las áreas de trabajo clínico; y (d) el desplazamiento de plazas en el sector público para contrataciones por servicios profesionales (Barreto \& López, 2013; García, Barreto \& Cruz, 2013). Todas estas circunstancias inciden en la profesión y a su vez, en la capacidad del gremio profesional de incidir sobre éstas. Sin embargo, dicha capacidad colectiva se verá mediada tanto por la condición asalariada y el contexto socio-histórico, así como por la comprensión/acción que el colectivo pueda tener/hacer respecto de las maneras en que como gremio profesional se enfrenta a los retos teórico-metodológicos, técnico-instrumentales y éticos-políticos de la actualidad. Para ello es necesario asumir, entre otros debates, a qué llamamos TSC, y cuáles retos nos presenta esta denominación en la actual coyuntura privatizadora del derecho a la salud.

\section{Dimensión terapéutica vs. TSC: algunas distinciones}

Reconocemos que la dimensión terapéutica del TS, (denominada por un sector profesional en Puerto Rico como TSC), tiene una larga trayectoria. Sin embargo, debemos distinguir entre estas dos formas de nombrar. Por un lado, "un profesional o una profesional del TS que ejerce una práctica

2 Voluntarios legos son aquellas personas que hacen trabajo voluntario en sustitución de profesionales aunque no cuentan con formación especializada o calificada en el área, pero que reciben entrenamiento para ejercer algunas funciones que generalmente desempeñan profesionales. 
clínica" y cuenta con competencias teórico-metodológicas, técnico-operativas y ético-políticas tanto para la intervención asistencial, socioeducativa-promocional y gerencial, como para la intervención terapéutica. Por otro lado, un profesional que bajo la categoría de "Trabajador Social Clínico" busca situarse en una práctica licenciada, privada, autónoma y liberal, cuya única o principal función es psicoterapéutica (Barreto, 2015). Este último, es un profesional que en Puerto Rico procura, por lo general, configurarse y legitimarse con su propia licencia profesional. Además, es un profesional con una funcionalidad distinta a la del TS en relación a la política social y el complejo médico-industrial (Barreto, 2012). Para comprender las distinciones que intentamos hacer entre un "profesional del TS con práctica clínica o terapéutica" y el profesional de "TSC" debemos referirnos a las definiciones y trayectoria de éste en el contexto de los Estados Unidos (EEUU).

Según Laura Groshong, una de las principales exponentes de esta práctica y directora de Política y Práctica del Clinical Social Work Association de los EEUU (CSWA), la definición de lo que es TSC es problemática. Groshong (2009) expone que la dificultad estriba en que los profesionales y las profesionales definen su identidad de diversas maneras, "a veces basados en los escenarios donde trabajan, algunas veces por las orientaciones teóricas que utilizan para comprender a sus clientes" (p. 2). Sin embargo, indica que la definición más generalizada (al menos en el 2009) era una provista por otra reconocida trabajadora social clínica, Eda G. Goldstein, quien la define como "la aplicación de los principios de salud mental al amplio ámbito del tratamiento de la salud mental" (según citado por Groshong, 2009). Por su parte, la National Association of Social Work de los EEUU avala una definición parecida a la elaborada en el año 2003 por Barker, aunque más específica: "la aplicación profesional de las teorías y métodos del TS 
para el diagnóstico, tratamiento y prevención de la disfunción, incapacidad o impedimento psicosocial, incluyendo trastornos emocionales, mentales y de conducta" (NASW, 2005, p. 9). Mientras, la American Board of Examiners in Clinical Social Work (ABE) define este ejercicio como:

Clinical social work is a mental-health profession whose practitioners, educated in social-work graduate schools and trained under supervision, master a distinctive body of knowledge and skill in order to assess, diagnose, and ameliorate problems, disorders, and conditions that interfere with healthy bio-psychosocial functioning of people-individuals, couples, families, groups-of all ages and backgrounds. (abecsw.org, Clinical Social Work Defined, s/f., parr. 1)

En los 50 estados de los EEUU se requiere una licencia particular para TSC que es diferente a la de TS, e incluso en algunos estados la única práctica para la cual se requiere una licencia y reválida es para la clínica (Association of Social Work Boards, 2013). En otras palabras, todas las demás dimensiones o prácticas del TS, entiéndase, gestión y administración, asistencia social, socio-educativa, comunitaria, de formulación y análisis de la política social, forense, escolar, entre otras, no requieren de licencias o reválidas particulares, pues son todas parte de lo que es el amplio campo del TS. Por esta razón, el ABE procura señalar que:

Recognized with their own title, Clinical Social Worker, and their own level of licensure (to protect the public) in every state in the union, Clinical Social Workers are mental healthcare professionals similar to clinical psychologists. While they are sometimes confused with other types of Social Worker, Clinical Social Workers 
are very different in their services and professional attainments, which include board-certification signifying competence in clinical knowledge and skills. (abecsw. org,Clinical Social Workers, s/f., parr. 3).

De esta manera se aseguran de especificar que un profesional de TSC es más parecido a un profesional de Psicología Clínica que a un profesional de TS. Sin embargo, la comparación entre estas dos profesiones es de carácter político y económico. Según Greenwood (2014) éstas se diferencian en cuanto a la preparación, la aplicación de pruebas psicométricas, la inclusión del contexto en la intervención y el salario recibido. Autores como Epple (2007), aseguran que el TSC se está ejerciendo sin la preparación especializada requerida, responsabilizando a los programas de formación de este hecho. Sin embargo, Cohen (2003) plantea que no existen estudios empíricos que hayan demostrado que el tratamiento ofrecido por una $u$ otra profesión es mejor. Por ello señala que, en el caso de los EEUU, profesionales de TSC se han convertido en una sustitución económica al tratamiento psicoterapéutico ofrecido por profesionales de la Psicología y la Psiquiatría. Para el autor, esto explica que en la actualidad quienes ejercen el TSC en los EEUU son los principales proveedores de servicios de salud mental.

En Puerto Rico, dada nuestra relación colonial y la exposición/ imposición al TS de los EEUU, se utilizan principalmente definiciones construidas en dicho país. $\quad Y$ al igual, como menciona Albite en el año 2010, se nombra la dimensión clínica con diversas nomenclaturas de acuerdo al lugar y el contexto en el cual se ejerce (según citado por Rosario \& Sánchez, 2012). En el año 2010, Rita Córdova, una de las principales exponentes del TSC en el país, publicó un libro sobre el tema. En éste, se hace evidente la influencia de Barker al ofrecer su 
definición como, "la aplicación profesional de la teoría del TS y del método para el tratamiento y la prevención de la disfunción psicosocial, la incapacidad, o el impedimento que incluye los trastornos mentales y emocionales", (p. 35-36). También Córdova, ofrece su versión autóctona al describirla como "práctica psicosocial de servicio directo con personas, familias y grupos que enfrentan situaciones/problemas vinculados a la salud mental" (según citada por Rosario \& Sánchez, 2012). Sin embargo, a pesar de que en muchas ocasiones se escucha hablar de un TS o un TSC autóctono o nacido en Puerto Rico, lo que se puede identificar en la profesión es el uso o la adaptación de modelos y acercamientos terapéuticos provenientes principalmente de los EEUU.

En Puerto Rico no existe una licencia particular para profesionales de TSC, aunque diversos grupos profesionales abogan para que se reconozca una licencia clínica. Por otra parte, se estima que los programas de maestría con especialidad clínica cuentan con el mayor número de estudiantes en el país.

En el 2012 hubo un intento de parte de la Junta Examinadora de Profesionales del TS de enmendar la ley reglamentadora de la profesión mediante el Proyecto del Senado 2627 para, entre otras acciones, crear una licencia de TSC, con su propio examen de reválida (Senado de Puerto Rico, 2012). Este esfuerzo contó con gran oposición y se convirtió en un movimiento promovido por el Colegio de Profesionales del Trabajo Social (Primera Hora, 2012). El proyecto fue derrotado, contando con la oposición de diversos sectores de la profesión a varias de las enmiendas propuestas (CPTSPR, 2012; Departamento de la Familia, 2012; Departamento de Justicia, 2012) o con críticas a la falta de participación de profesionales, organismos de la profesión e instituciones educativas en la redacción del mismo (CPTSPR, 2012; ANAETS, 2012). 
La resistencia a varias de las enmiendas a la ley, incluyó oposición a la creación de licencias por especialidad en las áreas clínica y forense. Se planteó la falta de claridad sobre lo que constituían las áreas de dominio de cada especialidad (CPTSPR, 2012; Alicea, 2012). De igual modo, la falta de disposiciones para prevenir posibles cesantías a profesionales que no tuvieran dichas licencias a pesar de contar con la preparación y la experiencia, impedir su comparecencia ante los tribunales o que no se contara con profesionales suficientes para satisfacer la demanda (Departamento de la Familia, 2012; Departamento de Justicia, 2012; Administración de Tribunales, 2012).

Carolina Rojas, (2008), trabajadora social costarricense y docente de la Escuela de TS de la Universidad de Costa Rica, define la dimensión terapéutica del TS como,

...los procesos de investigación-intervención realizados por un o una profesional, que pretenden lograr el cambio subjetivo, relacional y comunicativo de las y los sujetos, con el fin de que translaboren, resignifiquen y superen el sufrimiento subjetivo, o bien, que rompan con la repetitividad de su historia personal, familiar o comunitaria, cuando ésta se presenta como un obstáculo para su bienestar y el de su entorno. (Rojas, 2008, p.4).

En otro escrito, Rojas (2007a) señala que se debe evitar que la práctica terapéutica del TS se convierta en una práctica "psicologizante" que ignora los vínculos de las situaciones cotidianas particulares a estructuras sociales condicionantes y que despolitiza la intervención profesional al individualizar los problemas sociales. Para ello propone que, 
La intervención terapéutica, desde el TS, debe orientarse a que cada sujeto pueda percatarse de la repetitividad de su historia personal, familiar, comunal y social, debe abogar por la libertad, en el amplio sentido de la palabra, y en todo momento, debe tomar en cuenta los elementos económicos, sociales y políticos que subyacen en cada situación dolorosa que se experimenta a nivel individual. Esta posibilidad, implica necesariamente, un posicionamiento que rompe con la "psicologización" de la cuestión social. Esto a su vez puede lograrse si no se contrapone lo sociológico/ político y la atención psico-social en la práctica del TS, repolitizando la dimensión terapéutica y logrando insertar posibilidades transformadoras que surgen del develamiento de las complejas interacciones y tensiones entre lo social y la vida cotidiana de cada sujeto. (Rojas, 2007b, p. 57- 58).

Para Rojas, la práctica terapéutica tiene que ser una práctica politizada que devele las complejas relaciones entre la vida cotidiana y las estructuras condicionantes del orden socioeconómico-político-cultural. Además, aboga junto a Villegas para que las intervenciones terapéuticas se implementen,

[...] paralela y complementariamente con otras formas de trabajo profesional como la asistencial, la socioeducativo promocional y la de gestión, con el apoyo esencial que implica la investigación, que es realizada como sustento para la toma de decisiones en el proceso de atención. Esto implica que no se visualiza esta labor de forma aislada, sino como un complemento para responder a las múltiples necesidades que las personas presentan. (Rojas \& Villegas, s/f, p. 3)

Estas definiciones de Rojas \& Villegas coinciden con las expresadas por Lilian Albite en la Asamblea Anual del Colegio 
de Profesionales del TS de Puerto Rico de 2010, cuanto plantea que el "TSC",

[Es] una micropráctica dirigida a entender el sufrimiento humano en la experiencia de la vida cotidiana que impide que uno de sus bienestares, -sentirse bien, de sentirse realizado- y la consecución de sus metas y aspiraciones. $Y$, en ese sentido, la naturaleza terapéutica tiene un elemento de liberación, de emancipación, de apoyo, ¿verdad?, y de superación o translaboración, (como dicen algunos latinoamericanos), de los bloqueos, tanto al interior de las personas involucradas, cómo al exterior de los recursos sociales a la cuales están relacionados con el problema. Tienen bases metodológicas y teóricas diversas. (Albite, 2010, 266:275, según citado por Rosario \& Sánchez, 2012)

Finalmente, debemos mencionar que aunque Rojas reconoce que la dimensión terapéutica del TS ha sido denominada de diversas maneras (i.e. método de caso, trabajo de caso, tratamiento social, intervención individual, TS psiquiátrico y TSC, p. 55), tal cual es el caso en Puerto Rico, ella se refiere a ésta como "dimensión terapéutica", y no como TSC, como es costumbre aquí y en los EEUU.

Al comparar estas definiciones se hace evidente el carácter patológico y psicologizante que tiene el TSC aquí y en los EEUU. Mientras Rojas, Villegas y Albite elaboran una definición de la dimensión terapéutica del TS que procura reconocer la subjetividad de la persona como una de las dimensiones con la cuales se trabaja para procurar la propia comprensión del sujeto sobre los obstáculos, tanto personales como estructurales, que inciden sobre su propio bienestar, colocándolo "en las mediaciones de la historicidad del objeto y de la condición del sujeto según clase, género y otras" (Molina, 
2010, p.21). Además, Rojas y Villegas enfatizan que la terapia es una dimensión complementaria a otras que son igualmente necesarias si se quiere trabajar desde una perspectiva de derechos, de reconocimiento de la "cuestión social" y desde el ámbito de la acción socio-política.

\section{La salud mental en contexto}

Aunque es común hablar del problema de la salud mental del país, los datos actualizados para demostrar la prevalencia de la problemática no están accesibles. La publicación más reciente en la que se recopilan estadísticas sobre la salud mental en Puerto Rico es de 2012. En la misma se denuncia la falta de datos actualizados y se hace referencia a variedad de datos entre el 2000 y 2009. Sin embargo, concluyen que "más del 50\% de la población puertorriqueña tiene alguna situación que afecta su salud mental" (Rosario, Plaza, Báez \& Vera, 2012, p. 6). Más recientemente, un informe preparado por docentes e investigadores de la Universidad Carlos Albizu, señala que, "las desigualdades sociales y económicas, el desempleo, la criminalidad, la drogadicción, la falta de oportunidades y la violencia intrafamiliar son variables de riesgo para el desarrollo de trastornos mentales." (Martínez Taboas, et.al., 2014, Introducción). Por otra parte, se hacen evidentes los cuestionamientos acerca del exceso de diagnósticos, los diagnósticos tardíos, los nuevos diagnósticos incorporados al DSM que psicopatologizan conductas habituales y el uso excesivo de medicación para tratar situaciones que pudieran ser elaboradas a través de procesos terapéuticos e incluso, socio-educativos (Vale, 2013; Gómez, 2013; Ortiz, 2011). El DSM en particular, es cuestionado por los intereses económicos que se benefician de los discursos psiquiátricos 
y psicológicos y que funcionan como dispositivos de control disciplinario (Rivera-Pastrana, 2011; Vale, 2013). En la actualidad, el DSM ha adquirido mayor despliegue, uso y relevancia dentro de los discursos psiquiátricos y psicológicos y en las instancias de poder institucional y disciplinario. Y se coloca en el centro de la discusión la lucha entre el derecho a la salud como responsabilidad fundamental de las instituciones gubernamentales y las iniciativas de cuidado dirigido que procuran el control de gastos y la maximización de ganancias como incentivo operacional de las corporaciones de servicios de salud tales como hospitales privados y firmas aseguradoras (Fitzpatrick, 2013).

Comprender el estado de la salud mental requiere de un análisis complejo de esta, no solo en cuanto a factores biológicos, sino particularmente a factores socio-estructurales y discursivos. De entre éstos, nos interesa puntualizar la relación del fenómeno de la salud mental con la economía y el colonialismo, sus vínculos a manifestaciones de la "cuestión social", y los intereses económicos del complejo médico industrial ${ }^{3}$ en la generación de discursos, legislación y regulación de la salud mental.

Relación de la salud mental con la situación socio-económica y el colonialismo. La situación socio-económica de PR se reconoce en crisis. Según la Organización Mundial de la Salud la crisis económica es un factor que aumenta los problemas de salud mental y suicidios (Mundo Popular, 2009). Rosario \& Sánchez (2012) señalan que la situación socio-económica

3 El término complejo médico industrial se refiere al conglomerado de empresas multibillonarias en la industria de la salud. Esto incluye: hospitales, compañías de seguros, farmacéuticas, doctores y otros profesionales de la salud, suplidores de equipo, empresas de consultoría, empresas de construcción y de bienes raíces, firmas de contabilidad y bancos, entre otras. Este concepto fue originalmente acuñado en el 1971 con la publicación por Barbara Ehrenreich del libro The American Health Empire: Power, Profits, and Politics. (Estes, Harrington \& Pellow, s/f). 
tiene efectos en la salud física y emocional de la población en Puerto Rico. Situación que según el Dr. Ibrahim Pérez, "se ha querido atender desde un modelo de prestación de servicios sin considerar situaciones sociales que poseen como denominador común: la desigualdad económica entre las clases sociales" (según citado en El Nuevo Día, 2011, párr. 7).

Roche (2010) menciona que "uno de los efectos del colonialismo es afectar nuestra estima propia" (p. 33), refiriéndose a la estima del país. El autor atribuye que el colonialismo tiene efectos negativos en la salud emocional de la población puertorriqueña. Por otro lado, Córdova (2010), entiende que la subordinación al imperialismo ha tenido grandes efectos en la salud mental colectiva e individual. Colonialismo que afecta la vida social en la que hay una dependencia colectiva hacia el colonizador. Relacionando intrínsecamente el colonialismo con la salud mental en la población puertorriqueña.

Rivera (2010), argumenta que la salud mental es trastocada por factores económicos, sociales y políticos. Entiende como factor que afecta la salud mental la precarización de los servicios que se ofrecen tras la privatización de todo el sistema de salud, incluyendo los servicios de salud mental, en la década del '90. Por otra parte, el efecto del colonialismo impacta a la salud mental cuando se imponen leyes y formas de intervención creadas en los EEUU que no necesariamente responden al contexto socio-económico, político y cultural particular de Puerto Rico. Rivera, encontró en su investigación que las limitaciones económicas definían el acceso a los servicios que recibían las participantes y que dependiendo de la clase social a la cual pertenecía la persona que necesita de los servicios, así era la calidad y accesibilidad de los mismos.

Varas (2006), expresó que contemplar el tema de salud mental desde las características particulares del individuo 
fomenta una perspectiva individualista de esta situación. Contemplar esta temática desde el análisis del contexto social y su relación con la salud mental permitiría tener una visión más amplia sobre esta situación que es de índole económica. No sólo porque la crisis económica agudiza la misma, sino porque es el propio sistema económico donde se genera y perpetua. Este sistema no permite que se ofrezcan los servicios indispensables, ya que se procura mantener poca inversión para sostener el lucro de un sector económico y no necesariamente mejorar la calidad de vida de las personas en desigualdad. Tampoco se propician las condiciones para satisfacer las necesidades básicas necesarias para el bienestar y desarrollo integral de las personas, lo que pudiera reducir los problemas de salud de mental.

Por ende, es sumamente necesario mirar colectivamente la situación de la salud mental en el país. Nos encontramos en un sistema, el cual prefiere adjudicar las problemáticas sociales a la salud mental (aspectos psíquicos e individuales) antes de reconocer que muchas de éstas son producidas por las desigualdades que crean el propio sistema económico, político, social y cultural. Esta falta de reconocimiento está vinculada a mantener en pie el sistema capitalista-imperial, ya que las propuestas para atender esta situación implicarían transformaciones estructurales al orden económico capitalistacolonial.

\section{La salud mental como manifestación de la "cuestión} social". Las manifestaciones de la "cuestión social" en palabras de Arito (2001), son los "factores que influyen en la organización familiar, en la salud, en la educación, en la idea de justicia, determinan otros modos de relación e intercambio social, se constituyen en determinaciones operantes en la vida cotidiana de las personas" (pp. 3-4). Las manifestaciones de 
la "cuestión social" en la etapa neoliberal se reducen aún más a problemas sociales. Como diría Netto (1992), "la intervención estatal sobre la "cuestión social" se realiza (...) fragmentándola y parcializándola" (p. 22). Al punto que el Estado atiende las desigualdades que genera el capitalismo mediante la creación de políticas sociales sectoriales las cuales se enfocan en los llamados problemas sociales. lamamoto (como se cita en Aberbach, s.f) señala que,

las desigualdades pasan a ser vistas como "desvíos, que debensercontorneadosy controladosinstitucionalmente, según parámetros técnicos. Los conflictos sociales no son negados, pero lo que es expresión de lucha de clases se transforma en "problema social", materia prima de la asistencia. (p. 7)

Además, Aberbach (s.f.) añade que,

los factores vistos como problemáticos son transferidos de la estructura social para los individuos y grupos considerados como responsables por su ocurrencia. Consecuentemente, lo que debe ser cambiado son los hábitos, las actitudes y comportamientos de los individuos, teniendo en vista su ajuste social, contribuyendo así a remover "obstáculos" al "crecimiento económico. (p. 7)

He aquí entonces, que la "cuestión social" se expresa en el ámbito de la salud mental. Según Guadalupe (2004), el concepto de salud mental, no sólo es discutible sino que se dificulta definirlo ya que está cargado de aspectos valorativos. La salud mental o mejor dicho, lo que la sociedad entiende por este, se construye social e históricamente a través de discursos dominantes. La autora plantea que la salud mental está altamente vinculada al control social a través de los 
sujetos que la in-corporan. Foucault (1975), lo nombra como la docilización de los cuerpos.

Este autor plantea que, el control de los cuerpos se lleva a cabo a través de las instituciones. La locura fue tratada desde las instituciones del manicomio. Lo que en un inicio es considerado como un misterio de las divinidades pasó a ser, según Guadalupe, "un problema social debido a su "incapacidad" para acatar las normas impuestas y por no adaptarse a este nuevo orden social" (p. 33). Orden que se posibilita, no sólo con el control de la persona, sino que la misma persona es parte del proyecto de regulación social cuando hace funcionar las instituciones. Por tanto, la fragmentación que busca el orden capitalista, se hace palpable al tratarse la "cuestión social" como un asunto de salud mental. Así la "locura" se transforma en personas locas.

A pesar de que existe la lógica individual en la intervención profesional, es necesario reconocer la necesidad de ofrecer servicios de salud mental. Por esto, hay profesionales del TS insertados en proyectos o programas que ofrecen servicios a las personas que muestran problemas o dificultades con la salud mental. De esta forma, el o la profesional de TS vela por el derecho a la salud que tiene toda la población.

Para intervenir y trabajar con la psicologización de la vida social y con la salud mental, es necesario un profesional técnico. El o la profesional del "TSC" está posicionado en el trabajo más individual que se puede hacer con las personas -la psicologización de la salud mental. Por esta razón es imprescindible entender la relación entre la perspectiva psicologizante, la salud mental y el sistema socio-económico y político en el cual la profesión y su área clínica nacen, se desarrollan y se mantienen. Además, la comprensión total de esto permite analizar críticamente la formación y el quehacer profesional del "TSC". 
Los intereses del complejo médico industrial. El uso del término complejo médico industrial procura denunciar el negocio lucrativo que representan los servicios de salud y en el cual se incluye la inversión en la investigación y la educación en diversas áreas de la salud a fin de generar ganancias futuras en dicho campo. En la actualidad, el complejo médico-industrial es una de las principales áreas de inversión y ganancias en los EEUU, (Estes, Harrington \& Pellow, s/f). Además, el capital financiero ha logrado hegemonía en el control de este sector mediante la creación de organizaciones de cuidado dirigido (Iriart, 2008). Según Iriart (2008), las organizaciones de cuidado dirigido "son empresas de lucro creadas como subsidiarias por las empresas de seguro y por las administradoras de fondos mutuales y de pensión, para entrar en la administración de fondos de salud públicos y privados" (p. 1621). Dichas compañías, administran los riesgos de la inversión en la provisión de servicios de salud a través del manejo de servicios y el control de gastos. Para Cohen (2003), estas empresas aprovechan que el costo de contratación de profesionales del TSC es menor que el de profesionales de la psicología. Señala que las compañías de cuidado dirigido de la salud, han incidido en el aumento dramático de profesionales de TSC en el tratamiento de pacientes de salud mental, así como en las formas de intervención, refiriéndose principalmente a intervenciones basadas en teorías de la conducta o cognitivoconductuales, de terapia breve, y enfocadas en resolver síntomas agudos.

A partir de 1993 el sistema de salud pública en PR fue privatizado y la red de hospitales y clínicas de salud que ofrecían servicios de salud a toda la población a través de un sistema regionalizado, fue sustituida por un sistema de tarjeta de salud para personas de bajos ingresos que cualificaran para recibir la misma. La tarjeta de salud es provista por un 
puñado de compañías de seguros de salud que a su vez pagan por servicios a proveedores de salud a través de ocho regiones. En el caso de los servicios de salud mental, dichos servicios quedaron adscritos al Departamento de Salud y eran ofrecidos a través de la Administración de Servicios de Salud Mental y Contra la Adicción (ASSMCA). Sin embargo, en la actualidad la mayoría de estos servicios están siendo ofrecidos a través de APS, compañía contratada por la Administración de Seguros de Salud de PR (ASES) para ofrecer los servicios de salud mental y abuso de sustancias a las personas suscritas al seguro de salud del Gobierno de PR. Dicha compañía funciona con un modelo de Managed Behavioral Healthcare Organization (MBHO) o manejo de cuidado coordinado de la salud mental. APS es el tipo de compañía que bajo el discurso de "cuidado dirigido" coordina servicios de costo-efectividad que garantizan las ganancias a la empresa. En el caso de PR, las denuncias contra APS han estado ante la consideración del Senado tan recientes como en mayo del 2014,

Dilaciones en la otorgación de citas y la expedición de recargas de medicamentos, la denegación de servicios en las salas estabilizadoras, quejas por el trato a los pacientes, largo tiempo de espera para recibir servicios, deficiencia en la cantidad de actividades educativas ofrecidas, merma en tiempo de estadía por hospitalizaciones; son algunos problemas en el área de salud mental de la privatizadora APS Healthcare PR (APS) que quedaron expuestos durante una vista senatorial. (Noticel, 2014, parr. 1)

En la actualidad, profesionales de la salud mental, entre estos del campo del TS y de la Psicología Clínica, que por años ofrecieron sus servicios a través del Sistema de Salud Pública, han quedado a la merced de ser contratados por 
APS o de abrir oficinas privadas. Sin embargo, según Bernal (2006), la privatización de los servicios de salud mental no solo encareció la calidad de los servicios, en particular en el área de prevención, y el aumento los costos de la salud, sino que además señala que,

El impacto de la Reforma de Salud se hizo sentir en la práctica de la psicología con la desaparición de varios programas importantes e innovadores y con la devaluación del rol del psicólogo/a clínico con doctorado. De este modo, las empresas de cuidado coordinado emplearon a profesionales con menor preparación académica para ofrecer los servicios de tratamiento. (p. 368-369).

Aunque al presente, los y las profesionales de la psicología continúan siendo los principales proveedores de servicios de salud mental en el país, el complejo médico-industrial pudiera tener intereses en que se legitime la profesión de TSC para el ofrecimiento de servicios individualizados y por facturación, tal cual ocurre en los EEUU. Por otra parte, Barreto (2015) señala que,

El profesional con preparación de TS que tiene una oficina y practica exclusivamente la psicoterapia con individuos, parejas o familias y cobra por sus servicios directamente al cliente o a través de la facturación a un tercer pagador, (como son las aseguradoras de salud o el Medicaid), ejerce como terapeuta, no como trabajador/a social. Sobre todo, cuando para poder facturar un servicio a una aseguradora requiere de diagnosticar utilizando el DSM, y proceder con la aplicación de un modelo médico, que indiscutiblemente psicologiza la "cuestión social". Por ello que, en EEUU ni siquiera interesen identificarse como profesional de Trabajo Social. En este tipo de ejercicio la seguridad laboral está sujeta a la capacidad empresarial 
del o la profesional para hacer de lo que ofrece un negocio que le compense adecuadamente por su trabajo. (p. 11)

Por tanto, el problema del sistema de salud no es solo que focaliza la política social en el campo de salud mental a una cuestión que recae sobre el tratamiento, la medicación y la acción de disciplinar personas, desplazando toda posible responsabilidad sobre las estructuras que refractan la "cuestión social" a una "cuestión mental", sino que además, focaliza la práctica del TS al ejercicio exclusiva o prioritariamente clínico, pues las aseguradoras de salud no pagan por funciones sociopromocionales, asistenciales, gerenciales o de inserción en la política social. Se factura y se cobra exclusivamente por un diagnóstico médico basado en el uso del DSM y su tratamiento, preferiblemente de corta duración.

Sin embargo, ante los intereses de las aseguradoras de salud, las farmacéuticas y las compañías de cuidado dirigido se debe reconocer los esfuerzos de aquellos sectores y movimientos sociales que defienden el derecho a la salud y denuncian los intereses mercantilistas del complejo médicoindustrial. Incluso, el propio sistema de lucro en la salud es reconocido por varios sectores como parte intrínseca del problema de la salud en Puerto Rico, y de la salud mental en particular. Esto ha llevado a la creación de alianzas como la Alianza de Salud para el Pueblo, la cual favorece un sistema de salud universal con pagador único, iniciativa que es apoyada por el Colegio de Profesionales del TS.

\section{Problematizar el "TSC"}

En Puerto Rico, por nuestra relación colonial, se tiene una fuerte exposición/imposición de las formas como se ha construido la profesión en los EEUU. La política social ha 
cumplido funciones políticas que no solo legitiman la relación capital-trabajo en el capitalismo imperialista, sino además la propia relación colonial con los EEUU. En este contexto, el TS nace y se desarrolla en la dinámica del nuevo trato "americano" y su versión criolla, y más adelante en el marco de la política neoliberal.

Al mismo tiempo, un sector del colectivo profesional, generalmente vinculado a la academia y/o al independentismo, ha resistido o maniobrado, con diversos grados de éxito, esta exposición/imposición. En el caso del TSC, esta práctica ha sido criticada por diversas razones, bien por no ser considerada TS o por mercantilizar el TS. Hoy nos tocaría analizar el contexto actual en el que se impulsa esa profesión y sus implicaciones para el TS en PR.

Barreto (2012), siguiendo una perspectiva histórico-crítica acerca de la génesis del TS y la propuesta de Montaño y otros autores de Brasil de que el TS no es una evolución de la caridad y la filantropía, sino una ruptura que se da en el momento en que el Estado toma para sí la respuesta a la "cuestión social" en la fase monopólica del capitalismo, ha planteado que,

EI TSC-Privado (tal cual es ejercido en los EEUU) es una nueva ruptura, (y no una evolución del TS de caso), que se prolifera en respuesta a las nuevas configuraciones de la "cuestión social" en el marco del desmantelamiento del Estado de Bienestar, el neoliberalismo y la apertura del mercado de la industria de la salud de un servicio que, en el caso de los EEUU, es mucho menos costoso que pagar a terapeutas de la psicología o psiquiatría. Esta ruptura propicia que tal profesión se consolide en tal contexto como profesión liberal, estatus que el TS (no-clínico) no podrá alcanzar, por su vinculación directa con el Estado y las políticas sociales, principalmente como trabajador asalariado. (p. 8) 
Si la profesión es entendida como una que evoluciona y se profesionaliza en virtud de las respuestas de los y las profesionales a las "necesidades" de la población con la cual trabaja, el TSC sería considerado como el desarrollo razonable de la profesión ante los crecientes "problemas" de salud mental de la población. Sin embargo, si la profesión es entendida como un producto histórico cuya funcionalidad se vincula a la implantación de las políticas sociales, entonces el TSC, en la medida en que es "independizado, individualizado, liberalizado, psicologizado y privatizado" rompe como profesión con dicha base fundacional. A esto llamamos el ejercicio liberal de la profesión.

EI TSC en los EEUU es la forma de práctica principal en la cual se ejerce la práctica privada. La posibilidad de facturar por servicios psicoterapéuticos le ofrece a dicho profesional la oportunidad de establecer una oficina privada o de ser contratado por empresas o agencias que ofrecen servicios psicoterapéuticos y cobran mediante un sistema de facturación ya sea directamente al cliente o un tercer pagador (seguro de salud o Medicare y Medicaid). El hecho de que en dicho país las licencias en TS son para la práctica generalista, o para la práctica clínica o independiente, implica una ruptura entre lo que es considerado como categoría clínica y propia del ámbito privado y todo lo demás que pueda ser considerado como TS. En este sentido, el vínculo del TS con la política social como derecho social, es sustituido por un vínculo mercantilista con una política social como servicio de consumo en el mercado. Como consecuencia el proyecto ético-político del colectivo profesional, fundamentado en la defensa, exigibilidad y ampliación de los derechos humanos, la formulación de políticas sociales universales de calidad y accesibles, la democratización de los servicios sociales y el mejoramiento de las condiciones laborales del profesional de TS, se quiebra. 
Así mismo se encarecen, subestiman y estigmatizan las funciones asistencial y socio-promocional. En su lugar, prevalece la educación, la reválida y el licenciamiento clínico, la oferta de servicios individualizados, la competencia entre profesionales y la relación clientelar con las/os consumidores/ as. El proyecto del colectivo profesional se enfocaría entonces en promover la educación para el ejercicio clínico y la práctica privada o independiente, el reclamo de espacios para el ejercicio profesional en escenarios de salud, la demanda por equiparar la compensación por servicios psicoterapéuticos a la de profesionales de la psicología y la psiquiatría y el reconocimiento del TSC como una profesión equivalente y de igual reconocimiento y prestigio que la psicología clínica. En dicho escenario, la transformación social queda reducida al ámbito de las acciones individuales de los y las profesionales en su carácter personal y no a un proyecto profesional vinculado a los movimientos sociales por la defensa y ampliación de los derechos sociales.

\section{Reflexiones Finales}

Este trabajo no está en contra de la práctica clínica, ni de quienes ejercen como terapeutas. Es una problematización de lo que se denomina como TSC, particularmente lo que este representa para un proyecto profesional crítico. La práctica del TSC en su modalidad liberal, por su propia naturaleza, tiene que responder a la lógica del mercado y del empresarismo, pues de otra manera no podría subsistir. Más importante aún, aunque hayan profesionales con la disposición de ofrecer servicios ad honoren, o por cobro escalonado de acuerdo a los ingresos, se vincula el gesto a uno de caridad y filantropía. Sostenemos que, quien ejerce el Trabajo Social como profesión tiene el 
deber y la responsabilidad de ofrecer el servicio en virtud de un derecho social. Particularmente, en un momento en el cual la política neoliberal procura colocar la responsabilidad de las manifestaciones de la "cuestión social" en la propia persona, mientras el complejo médico industrial empuja a que así sea para su propia ganancia económica.

A esto se suma, que la acción terapéutica se ofrezca aislada de otras formas necesarias de acción profesional, haciendo irremediable que dicha práctica sea una eminentemente psicologizante. Incluso sin obviar que otras dimensiones de la práctica profesional pueden ser igualmente psicologizantes en la medida que parten de un acercamiento teórico-metodológico positivista y funcionalista, pues la diferencia está en sus fundamentos filosóficos y teórico-metodológicos. La riqueza interdisciplinaria, multi-módica, inter/multidimensional del TS se reduce a la acción terapéutica, la cual desde acercamientos como los cognitivo-conductuales encuentran tanto el problema como su solución en el individuo. Así, en un escenario escolar, por ejemplo, el problema del bulling se enfoca en la víctima y el victimario y no en las relaciones de poder de la comunidad escolar; la llamada deserción escolar se enfoca en las faltas del estudiante y su familia, y no en las carencias de un sistema escolar que expulsa estudiantes para caer en las arcas de las empresas educativas (con y sin fines de lucro); y las problemáticas de la niñez con necesidades especiales se enfoca en pagar la multa por incumplimiento, y no en los intereses del complejo médico-industrial de aumentar la medicalización y los diagnósticos de salud mental de nuestra niñez a cifras altamente cuestionables (Barreto, 2015).

Un TS de carácter crítico y propositivo debe procurar una fuerte organización del gremio profesional para incidir en los asuntos de política social, en particular un sistema 
de salud universal, de calidad y accesible, y en la denuncia de los intereses económicos que están detrás de la contrareforma del Estado, la precarización de la política social y de los servicios sociales, así como el empeoramiento de nuestras condiciones laborales. Debe, además, reconocer la terapia como complementaria a otras dimensiones de la profesión. Sin embargo, debe demandar que esta sea ejercida en virtud de políticas sociales universales, exequibles y de calidad, en contextos inter/multi/transdisciplinarios, desde escenarios públicos e incluso privados y teniendo como referente la resignificación de la experiencia subjetiva, relacional y comunicativa de los sujetos, a fin de potenciar su salud y autonomía crítica, y ofrecidas de manera complementaria e integral a las dimensiones asistencial, socio-promocional y de inserción en la policía social y la investigación. Tendría además que, pronunciarse en contra de una licencia particular en TSC que fragmente la profesión, de una práctica alienada que solo factura y cobra por el ejercicio de la psicoterapia, y del uso del DSM y el modelo médico para diagnosticar, etiquetar, facturar y psicologizar la "cuestión social", colocando la responsabilidad en el sujeto de las manifestaciones de la "cuestión social" que le vulneran.

La conciencia clara de estos desafíos, y la discusión y análisis complejo de los mismos es indispensable para ampliar la base de nuestro poder como colectivo profesional. Sin embargo, ignorar la necesaria discusión de estos asuntos o discutir solo pensando desde los intereses personales debilitan nuestra capacidad de respuesta como colectivo ante los desarrollos del proyecto neoliberal y sus implicaciones para la profesión de TS en PR. 


\section{Referencias:}

Aberbach, Graciela. (s.f.). ¿Los argentinos estamos locos? Recuperado de http://www.ts.ucr.ac.cr/binarios/pela/ pl-000065.pdf

Administración de Tribunales (2012). Ponencia ante la comisión de Gobierno del Senado de Puerto Rico Sabado, 23 de junio de 2012 Proyecto del Senado 2627 (2012). Puerto Rico.

Alicea Rodríguez, Larry (2012). Memorial sobre el Proyecto del Senado 2627 Ley de Profesionales en Trabajo Social Siglo $X X \mid$. Puerto Rico.

American Board of Examiners in Clinical Social Work (s.f). Clinical Social Workers. Recuperado el 13 de abril de 2015 a través de https://www.abecsw.org/csw-marketplace.html

ANAETS (5 de diciembere de 2012). Comunicado de Prensa: P. del S. 2617, Asociación Nacional de Escuelas de Trabajo Social. Recuperado de: https://anaetspr.org/proyecto-delsenado-2627/

Arito, S. (2001). Cuestión social y salud mental: una relación implicada en la intervención profesión. Recuperado de www.ts.ucr.ac.cr.

Association of Social Work Boards (2013). Table 2: Levels of Practice Regulated. Recuperado a través de: https:// www.datapathdesign.com/ASWB/Laws/Prod/cgi-bin/ LawWebRpts2DLL.dll/Osv7gx00n5wu5c1b6j7py084axpr/\$

Barreto Cortez, Esterla (2012). Los desafíos para el TS de una especialidad clínica en el contexto de un proyecto social neoliberal: una encrucijada para el proyecto ético-político profesional. Ponencia presentada en el XX Seminario Latinoamericano de Escuelas de TS, Córdoba, Argentina. 
Barreto Cortez, Esterla (2015). Precarización de la condición laboral y psicologización de la "cuestión social" en el contexto de la salud como negocio: desafíos para el proyecto ético-político del TS en PR. Sometido para publicación a la Revista "Praia Vermelha".

Barreto, Esterla \& Lopez, Mabel. (2013). Informe sobre estudio de las condiciones laborales 2011. Colegio de Profesionales del Trabajo Social de Puerto Rico, CPTSPR.

Bernal, Guillermo (2006). La psicología clínica en PR. En Revista Puertorriqueña de Psicología, 17, 341:388

Clínica Internacional de Derechos Humanos de la Facultad de Derecho de la Universidad Interamericana de Puerto Rico \& Instituto Caribeño de Derechos Humanos (4 de abril de 2016) Informe presentado ante la Comisión Interamericana de Derechos Humanos Sobre: Deuda Pública, Política Fiscal y Pobreza en Puerto Rico.

Cohen, Jefrey A. (2003). Managed Care and the Evolving Role of the Clinical Social Worker in Mental Health. En Social Work, Revista de la National Association of Social Workers, Inc., 48 (1) 34:43.

Córdova, Rita. (2010). Trabajo Social Clínico en PR: Construcción de la personalidad puertorriqueña. San Juan, PR: Action Printing.

Cotto Morales, Liliana (2007) Soñando alternativas para una política de vivienda en tiempos de desesperanza. En Guardiola Ortiz, Guemárez Cruz y Rivera Casiano (Eds.), La crisis sostenida: retos para la política social y el trabajo social. (341-364). San Juan, PR: Edtorial Edil.

CPTSPR (2012). Memorial explicativo proyecto del Senado 2627. Puerto Rico. 
Departamento de Justicia. (2012). Proyecto del Senado 2627. Puerto Rico.

Departamento de Salud (s.f.). Salud Mental: Análisis de Situación de la Salud en Puerto Rico. Recuperado a través de: https:// www.salud.gov.pr/Documents/Acreditaci\%C3\%B3n\%20 del\%20Departamento\%20de\%20Salud/PRDoH\%20 CHSA\%20(Draft\%20Art)\%20Salud\%20Mental.pdf

Díaz Coss, Josué R., López Nieves, Cruz A. Pagán Rivera, Víctor G. (2015). La precarización del trabajo social a través del voluntariado social: nuevos modos sociales de regulación en la relación capital-trabajo en el contexto puertorriqueño, 2014-15. Tesis presentada a la Escuela Graduada de Trabajo Social de la Universidad de Puerto Rico. Recinto de Río Piedras.

El Nuevo Día (14 de mayo de 2014) La sobremedicación de ancianos. Recuperado a través de: http:// www.elnuevodia.com/estilosdevida/hogar/nota/ lasobremedicaciondeancianos-1772578/

El Nuevo Día (26 de septiembre de 2011). "Laguna" en la salud mental. El Nuevo Día. Recuperado de https://www.adendi. com/archivo.asp?Xnum=1076333\&year=2011\&mon=9

Epple, Dorothea Marie (2007). Inter and Intra Professional Social Work Differences: Social Works Challenge. En Clinical Social Work Journal, 35:267-276. DOI 10.1007/ s10615-007-0098-0

Estes, Carroll L.; Harrington, Charlene \& Pellow, David N. (2010). Medical-Industrial Complex. En Borgatta and Montgomery Encyclopedia of Sociology, Second Edition. Recuperado en: edu.learnsoc.org 
Fitzpatrick Usera, Mario (2013). La influencia neoliberal en los servicios de salud mental privado de PR. (Tesis de maestría no publicada). Centro de Estudios Avanzados de PR y el Caribe.

Foucault, M. (1975). Vigilar y castigar: nacimiento de la prisión (14a. ed.). México: Siglo XXI.

García Toro, Víctor Iván, Barreto Cortez, Esterla y Cruz Soto, Aurea (2013). Avanzar al pasado: la des-profesionalización del Trabajo Social en Puerto Rico. En Estudio: Condiciones Laborales de los y las Profesionales de Trabajo Social, noviembre 2011. Informe final de la Comisión Permanente del Proyecto Profesional del Colegio de Profesionales del Trabajo Social de Puerto Rico.

Gómez, Antonio R. (28 de octubre de 2016) Muchos niños mal diagnosticados en educación especial. En Primera Hora. Recuperado a través de: http:// www.primerahora.com/noticias/puerto-rico/nota/ muchosninosmaldiagnosticadoseneducacionespecial $-1044479 /$

Gómez, María De Los Ángeles (7 de noviembre de 2014). La priva(tiza)ción de la salud mental. En 80 Grados. Recuperado a través de: http://www.80grados.net/la-privatizacion-dela-salud-mental/

Gómez, María de los Ángeles (8 de noviembre de 2013). La batalla de los diagnósticos. En 80grados.net Recurado a través deL www.80grados.net/la-batalla-de-los-diagnosticos/

Greenwood, Beth (27-nov-2014). Social Work Vs. Clinical Psychology. En Chron Media. Recuperado de: http://work. chron.com/social-work-vs-clinical-psychology-6672.html

Groshong, Laura W. (2009). Clinical Social Work Practice - An overview. Lanham, MD: University Press of America. 
Iriart, Celia(2014) Medicalización, biomedicalización y proceso salud-enfermedad-atención. Ponencia presentada en la XI Jornadas Nacionales de Debate Interdisciplinario en Salud y Población, Agosto 2014. Recuperado a través de: http://jornadassaludypoblacion.sociales.uba. ar/files/2014/11//riart_Celia.pdf

Iriart, Celia. (2008). Capital financiero versus complejo médicoindustrial: los desafíos de las agencias regulatorias. En Ciência \& Saúde Coletiva, 13(5):1619-1626.

Mario Fitzpatrick Usera. (2015). Del Asilo de Insanos a Wall Street. San Juan: Editorial Trans. Biblio Services

Martínez-Taboas, Alfonso; Hernández, Héctor; Rodríguez Gómez, José; Lynn Suarez, Joy; Nazario-Serrano, Juan A.; \& Narvaez. Karla. (2014). Estado de situación de la salud mental en PR. Universidad Carlos Albizu.

Maria Lorena Molina (2010). Hacia una Intervencion Profesional Critica en Trabajo Social Hacia una Intervencion Profesional Critica en Trabajo Social. Argentina: Espacio Editorial.

Montaño, Carlos. (1998). La naturaleza del servicio social: un ensayo sobre su génesis, su especificidad y su reproducción. Brasil: Cortez Editora.

Mundo Popular. (2009, 5 de junio). Crisis económica aumenta las enfermedades mentales. Recuperado de http:// www.mundopopular.org/crisis-econ-mica-aumenta-lasenfermedades-mentales/

National Association of Social Work. (2005). NASW Standars for Clinical Social Work in Social Work Practice. Recuperado de http://www.naswdc.org/practice/ standards/NASWClinicalSWStandards.pdf 
Netto, José Paulo \& Braz, Mauricio (2007). Economía Política: Una introducción crítica. Biblioteca Básica de TS, vol. 1. São Paulo: Cortez Editora.

Netto, Jose Paulo. (1992). Capitalismo Monopolista y Servicio Social. Brasil: Cortez Editora.

Northen, H. (1995). Evolution of clinical social work. En Clinical Social Work: Knowledge and skills (pp.1-15). New York: Columbia University Press.

Noticel. (2014, 5 de abril). Larga lista de quejas contra APS, privatizadora de servicios de salud mental. Recuperdo de: http://www.noticel.com/noticia/158395/larga-lista-dequejas-contra-aps-privatizadora-de-servicios-de-saludmental.html

Ortiz Roselló, Rosalyn I. (2011). El DSM-5 y su estrategia de psicopatologizar la vejez. En Cruce, 1.

Pérez Soler, Ángel (10 de julio de 2015) Lobos vestidos de ovejas: La privatización de los servicios sociales. En 80 Grados. Recuperado a través de: 80grados.net/ lobos-vestidos-de-ovejas-la-privatizacion-de-los-serviciossociales/

Primera Hora (14 de mayo de 2012). Trabajadores Sociales se oponen a proyecto. Recuperado de: http:// www.primerahora.com/noticias/puerto-rico/nota/ trabajadoressocialesseoponenaproyecto-647023/

Rivera Díaz, Marinilda. (2014). Ciudadanos invisibles: niñez, política y salud mental en Puerto Rico. San Juan, P.R.: Centro de Investigaciones Sociales, Universidad de Puerto Rico. 
Rivera, Marinilda (2010). Ciudadanos/as invisibles: Diversidad de voces sobre la política de salud mental en Puerto Rico. (Disertación doctoral no publicada). Universidad de Puerto Rico, Recinto de Río Piedras, San Juan, PR.

Rivera-Pastrana, Luz Ivette (2011). Cuerpos dóciles y cuerpos insubordinados: Deconstrucción del trastorno disfórico premenstrual. Dissertation Abstract, Programa de Psicología de la Universidad de PR. Document URL: http://search. proquest.com/docview/888562674

Roche, Mario (2010). Trabajo Social y el status político de Puerto Rico. Revista de Servicio Social, 1(1), 33-42.

Rojas Carolina. y Villegas, César (s/f). Desafiando mitos: consideraciones sobre la legitimidad de los procesos terapéuticos desarrollados en TS. Material sin publicar.

Rojas Madrigal, Carolina (2007a). La dimensión terapéutica del Trabajo Social y la protección de los derechos humanos. Ponencia presentada en IV Congreso Internacional - VII Congreso Nacional de Trabajo Social San José, Costa Rica, 5 a 7 de setiembre de 2007.

Rojas Madrigal, Carolina (2007b). Dimensión Terapéutica del Trabajo Social Costarricense: un Análisis de su Surgimiento y Desarrollo en Cuatro Instituciones Pioneras. Tesis sometida a la consideración de la Comisión del Programa de Estudios de Posgrado en Trabajo Social para optar al grado de Magistra Scientiae en Trabajo Social con énfasis en Intervención Terapéutica. Sistema de Estudio de Posgrado, Universidad de Costa Rica, Sede Rodrigo Facio.

Rojas Madrigal, Carolina (2008). ¿Por qué surge y se desarrolla la dimensión terapéutica en el TS costarricense? En Revista Costarricense de Trabajo Social. (20). 
Rosario Nieves, llia; Plana Montero, Ana \& Báez Rivera Arvin (2012). La Salud Mental en Puerto Rico: una mirada desde las estadísticas y las políticas públicas. Cuadernos de Investigación Social y Económica, Pontificia Universidad de Puerto Rico. Recuperado de: http://www.consejeria.net/ uploads/3/4/1/6/34163570/estudio_la_salud_mental_en_ pr.pdf

Rosario-Rivera, Nélida \& Sánchez-Marcano, Francine. (2012). Trabajo Social Clínico: discursos sobre las prácticas profesionales y formativas del ejercicio clínico en el contexto socio-histórico y político de Puerto Rico. (Tesis de maestría no publicada). Universidad de Puerto Rico, Recinto de Río Piedras.

Rosario-Rivera, Nélida \& Sánchez-Marcano, Francine. (2011). Práctica y formación del Trabajo Social Clínico en el contexto socio-económico y político de Puerto Rico. Ponencia presentada en el III Congreso Nacional de TS y II Encuentro Latinoamericano de Docentes, Profesionales y Estudiantes de TS, Argentina.

Senado de Puerto Rico (2012). Proyecto del Senado 2627 para añadir un nuevo Artículo 1; renominar los Artículos 1 al 22 como los Artículos del 2 al 23; enmendar los Artículos $1,5,6,7,8,9,10,11,12,18$ de la Ley Núm. 171 de 11 de Mayo 1940, según enmendada, conocida como "Ley de Colegiación de los Trabajadores Sociales", a los fines de denominar esta ley como "Ley de Profesionales en Trabajo Social siglo XXI". Recuperado de: http://www.oslpr.org/ legislatura/tl2009/tl_busca_avanzada.asp 
Silva Gotay, Samuel (18 de Junio de 2011) Hacia una explicación de la destrucción del Estado en Puerto Rico a manos de la dirección del PNP por vía de la política neoliberal. Ponencia presentada a la Mesa de Diálogo Martin Luther King. Recuperado a través de: http://www.mesadedialogomlk. org/documentos/PNP.pdf

Torres González, Roamé. (1 de octubre de 2015) Ponencia ante la "Comisión Especial para la Transformación Administrativa del Sistema Educativo de Puerto Rico" sobre el Proyecto del Senado 1456 que busca crear la Ley para las Alianzas de en la Educación Pública del Estado Libre Asociado de Puerto Rico. Recuperado a través de: http://www.oslpr. org/2013-2016/ponencias/A3B2PNJ0.pdf

Torres Rivera, Alejandro. (10 de noviembre de 2011). Las modificaciones neoliberales de la política laboral en Puerto Rico: su origen y su impacto en las relaciones obrero patronales. Ponencia presentada ante la Septuagésima Segunda Asamblea Anual del Colegio de Profesionales del Trabajo Social de Puerto Rico, Transformaciones en los escenarios laborales del Trabajo Social: reflexiones y análisis. Ponce, PR.

Vale Nieves, Otomie (2013). El DSM como mecanismo de psicopatologización y regulación social: el caso de los niños/as en PR. En Quaderns de Psicología, 15(1): 21-32. Recuperado a través de: http://www.quadernsdepsicologia. cat/article/viewFile/1164/877

Varas, N. (2006). Saludmental en contexto. Asociación Psicología de Puerto Rico. Recuperado de: http://www.asppr.net/ index. php?view $=$ article\&catid $=910$ : articulos\&id $=58$ : saludmental-en-contexto\&option=com_content\&ltemid=69 